\title{
Competition strategies and correlated selection on responses to polyandry in the seed beetle Callosobruchus maculatus
}

\author{
R A U L N A R C I S O C. G UEDES ${ }^{1,2}$ and R O B E R T . S M I T H ${ }^{2,3}$ \\ ${ }^{1}$ Departamento de Biologia Animal, Universidade Federal de Viçosa, Viçosa, MG, Brazil, ${ }^{2}$ Department of Biology, University of \\ Leicester, Leicester, U.K. and ${ }^{3}$ School of Applied Sciences, University of Huddersfield, Queensgate, Huddersfield, U.K.
}

\begin{abstract}
Polyandry reflected in multiple mating with different mates is regarded as favoured by natural selection in males but not necessarily in females, where conflicting effects on fitness components can occur. The present study aims to provide empirical evidence to predict which fitness components may be affected in this sexual conflict using a species that demonstrates potential between-population variation in their resolution: the cowpea weevil Callosobruchus maculatus. Two strains showing contrasting competition outcomes (scramble $\times$ contest) and contrasting life-history strategies based on trade-offs between longevity and fecundity are crossed for subsequent selection based on larval-competition strategy, expecting the production of a correlated response to multiple (polyandrous) mating. Such a response is expected because the scramble strain shows high fecundity (and lower longevity) and would benefit from multiple mating, in contrast with the contest strain, which shows high juvenile mortality. The scramble-selected lines would evolve a response of increased fecundity and reduced longevity under multiple and potentially polyandrous mating but the contest-selected lines would not respond to multiple (polyandrous) mating. Instead, both scramble- and contest-selected lines show increased fecundity and reduced longevity with multiple (polyandrous) matings, which did not affect egg weight. Indirect benefits of multiple (polyandrous) mating appear to be relevant for lines showing contest competition among juveniles.
\end{abstract}

Key words. Bruchidae, contest competition, fecundity-longevity trade-off, multiple mating, scramble competition.

\section{Introduction}

Transfer of sperm from male to female is the fundamental role of mating but the evolution of anisogamy leading to sex role differentiation also leads to differential investments in offspring by the two sexes. This takes place because sexually reproducing organisms are under evolutionary selection pressure to maximize their fitness but adaptations to achieve this in one sex can reduce or constrain fitness in the other: a sexual conflict (Parker, 1979, 2006). Males maximize their fitness by mating with multiple mates, whereas females

Correspondence: Raul Narciso C. Guedes, Departamento de Biologia Animal, Universidade Federal de Viçosa, Viçosa, MG 36571-000, Brazil. Tel: +55 313899 4008; fax: +55 313899 4012; e-mail: guedes@ufv.br maximize theirs by maximizing the number of viable offspring produced, which may require only one or few matings (Parker, 1979, 2006; Jennions \& Petrie, 2000; Hosken \& Stockley, 2003; Tregenza et al., 2006). Nonetheless, females of many, if not most, taxa do copulate with multiple mates (polyandry), particularly among insects (Arnqvist \& Nilsson, 2000; Hosken \& Stockley, 2003; Arnqvist et al., 2005).

Polyandry is widespread despite its associated costs, which include the general expenses of time and energy among others (e.g. increased predation risk, physical injury and parasite/pathogen infection) (Arnqvist \& Nilsson, 2000; Johnstone \& Keller, 2000; Lessells, 2005, 2006). Therefore, the benefits of polyandry should outweigh its costs for it to prevail (Arnqvist \& Nilsson, 2000; Lessells, 2006). Such benefits may directly affect female fitness (e.g. either benefiting the female herself or her offspring number) or may provide indirect 
genetic benefits increasing offspring fitness (Yasui, 1998; Vahed, 1998; Jennions \& Petrie, 2000; Hosken \& Stockley, 2003). Current evidence of genetic benefits associated with polyandry exists but such benefits do not appear to be sufficiently large to offset the large direct costs incurred by females (Hosken \& Stockley, 2003; Tregenza et al., 2006).

Invertebrate taxa with presumed high rates of mortality and high reproductive output frequently exhibit an increased female lifespan and/or fecundity as benefits of polyandry (Hosken \& Stockley, 2003). However, there is a range of effects of multiple mating, or more specifically of polyandry, in females of different species and no general theory of how polyandry trade-offs might evolve, for example, between increased fecundity and reduced longevity. One way of resolving this shortcoming would involve the identification and study of species that demonstrate between-population variation in the resolution of these trade-offs.

Simple life-history theory shows how the optimal balance of reproduction versus adult longevity is determined by juvenile survival (Sibly \& Calow, 1986; Smith, 1991). High juvenile survival favours high fecundity and low adult longevity, and vice-versa. In animals whose juvenile stages exploit patches of resources (e.g. seed predators, parasitoids), juvenile survival is determined by the form of juvenile competition, broadly characterized as 'scramble' or 'contest' (Nicholson, 1954). The cowpea weevil Callosobruchus maculatus F. (Coleoptera: Bruchidae) is a species of seed beetle with different biotypes or strains distributed worldwide (Guedes et al., 2003; Messina, 2004), a likely result of the discontinuous nature of the stored seed environment and periodic inbreeding in this species (Tran \& Credland, 1995). Larvae of this species feed and develop within the seed on which the female parent laid eggs.

Two strains of $C$. maculatus, one from India and one from Brazil, have evolved intrinsically different larval competition strategies, as is modelled by Smith \& Lessells (1985) and Smith (1990). Larvae of the Indian strain compete aggressively within a seed such that only few individuals survive (contest competition), whereas Brazilian larvae avoid direct contact and several may emerge from a seed (scramble competition) (Guedes et al., 2007). Broadhurst (1996) show that it is possible to hybridize the two strains, and then select for pure contest or scramble lines over a few generations. These two strains evolve contrasting life-history strategies based on trade-offs between fitness components of longevity and fecundity, in accordance with life-history theory. There is a trade-off between fecundity and longevity, such that high juvenile survival leads to high fecundity and low adult longevity in the Brazilian strain, whereas low juvenile survival leads to the converse in the Indian strain (Smith, 1991).

The scramble strain with high juvenile survival from Brazil has fitness increased by higher fecundity, even if longevity is reduced (Guedes et al., 2007). By contrast, the contest strain with low juvenile survival from India would gain little benefit from increased fecundity (intense larval competition means that seeds rather than eggs are limiting) but has reduced fitness if longevity decreases (Smith, 1991). LobleyTaylor (2000) reports an investigation of how females respond to polyandry in the two strains in a preliminary attempt to characterize both strains regarding this response and report that the scramble strain (Brazil) responds with an increase in fecundity but a decrease in longevity, whereas the contest strain (India) shows no detectable response. Such a lack of response to polyandry is predicted by the life-history theory (Smith, 1991).

The direct female benefits of multiple polyandrous mating in $C$. maculatus are maximum sperm replenishment and provision of male ejaculates as nuptial gifts, besides eventual indirect genetic benefits, which should be higher for multiple polyandrous than for multiple monandrous mating (Wilson et al., 1999; Jennions \& Petrie, 2000; Hosken \& Stockley, 2003; Arnqvist et al., 2005; Yamane \& Miyatake, 2005). The main costs of multiple polyandrous mating for females of C. maculatus are egg production, male harassment and mating damage by males (Crudgington \& Siva-Jothy, 2000; Yanagi \& Miyatake, 2003; Arnqvist et al., 2005; Rönn et al., 2006). Polyandry may shift the balance between its costs and benefits in strains with contrasting life histories, probably favouring female fitness increase in scramble strains by plentiful provision of sperm and nutrients via male ejaculates. By contrast, no fitness increase is expected in multiple-mated females of contest strains because their offspring are subjected to higher mortality. In this case, sperm replenishment by multiple mating is not a benefit and increased male ejaculate alone does not seen able to compensate the costs of mating multiply, which therefore prevents fitness increase with multiple mating in these strains. Indirect (genetic) benefits of multiple polyandrous mating for females of contest strains may still take place but such benefits are usually regarded as being of secondary in importance for resolving sexual conflicts (Arnqvist \& Nilsson, 2000; Hosken \& Stockley, 2003; Tregenza et al., 2006).

The established features of the two strains of $C$. maculatus, Brazilian and Indian (contrasting life history, different responses to selection), make this species a potential model for providing empirical evidence to predict which fitness components may be affected in the sexual conflict underlying polyandry. It is expected that selection based on larvalcompetition strategy will produce a correlated response to exposure to polyandry. Specifically, scramble-selected lines will evolve a response to polyandry of increased fecundity and reduced longevity but contest-selected lines will not respond to polyandry, as preliminarily observed in parental strains by Lobley-Taylor (2000).

\section{Materials and methods}

\section{Insect populations}

Callosobruchus maculatus is a widely distributed pest of legumes. The adults show a short life span and females attach their eggs to beans, where the larvae develop inside. The two geographic strains used in the present study are the same as used in other recent studies (Lobley-Taylor, 2000; Fricke \& Arnqvist, 2004; Guedes et al., 2003, 2007). They have both been in culture for more than 25 years. The strain from Brazil 
was originally collected from cowpeas in Campinas in 1975, whereas the Indian strain was originally collected from mung beans and blackgram in Tirunelveli in 1979. The Brazilian strain was derived from stock cultures maintained at the U.K. National Resources Institute and the Indian strain was derived from stock cultures maintained at the Department of Biology, Utah State University (Logan, Utah). The Indian strain has been maintained on mung beans, unlike the Brazilian strain, which is maintained on cowpeas but new lines were established on cowpea for three generations before use in the present study. The insect cultures were maintained using the same procedures and densities in an environmentally controlled room, where the subsequent crosses, selection lines and experiments were carried out under an LD 12: $12 \mathrm{~h}$ photoperiod at $30 \pm 2^{\circ} \mathrm{C}$ and $70 \pm 10 \% \mathrm{RH}$.

\section{Crosses and selection protocol}

Seeds infested with a single larva (showing emergence windows) from the Brazilian and Indian strains were individualized in 25-well covered tissue culture plates to obtain virgin adults ( 1 day old) for the reciprocal crosses. One hundred pairs for each reciprocal cross were established to start both (unreplicated) $F_{1}$ lines: females from Brazil $\times$ males from India $\left[F_{1}(\mathrm{Br} \times \mathrm{In})\right]$ and females from India $\times$ males from Brazil $\left[F_{1}(\mathrm{In} \times \mathrm{Br})\right]$. Both reciprocal $F_{1}$ lines were subsequently combined into four replicated lines for the $F_{2}$ generation, whose identity and independence were maintained throughout the later selection regime. Each replicated line was again established upon individualization of seeds showing emergence windows to obtain virgin insects (1 day old) and using 50 pairs in $250 \mathrm{~g}$ of cowpea seeds (approximately 1200 seeds) to establish each of the four replicated lines. The pairs were able to interact freely until their eventual death. The four established lines were individually replicated each generation for three successive generations of relaxed selection. This period of relaxed selection was allowed to recognize the effect of natural selection on the traits under investigation.

The selection lines for scramble and contest competition were started after a three-generation period of relaxed selection using 200 cowpea seeds from each replicated line and individualizing them in wells of 25-well tissue culture plates. Each replicated line was independently selected (and maintained) for scramble or contest competition based on the number of insects emerging per seed containing over five eggs laid on its surface, within a 10-day period. The selection criteria were a maximum of two adults emerged per seed for contest competition and four or more insects emerged per seed for the scramble selection. The selection was always carried out on newly-emerged virgin adults (1 day old). The aim was to select at least 50 couples (placed in $250 \mathrm{~g}$ of cowpea seeds, as previously explained) for each line to start a new generation for further selection but, after the fourth generation of selection for contest competition, the new generations were established with 30-50 couples. Six generations of selection for scramble and contest competition were carried out.

\section{Protocol for single $\times$ multiple (polyandrous) mating}

The parental strains (i.e. Brazilian and Indian) and the derived lines were subjected to two mating systems. Females were either single mated or multiple mated when successively exposed to different males. Female weight, lifetime fecundity (number of eggs laid per female until her natural death), longevity, egg weight and proportion of eggs laid alone on the seed (singleton eggs) during the first day of the female's fertile life were assessed using 20-25 females for each combination of line (i.e. scramble and contest) and mating system (i.e. single or multiple polyandrous mating). A minimum of five females of each of the four independent replicated lines of selection (i.e. at least 20 females for each selection system: scramble or contest) was used (four for scramble and four for contest competition). Each replicated line of selection was individually maintained throughout the experiment without mixing. The protocol used for single and multiple mating was adapted from Lobley-Taylor (2000).

Virgin females (24-48 h old) obtained from individualized seeds were weighed in a Cahn electrobalance and allowed to mate with virgin males (also $24-48 \mathrm{~h}$ old). Each female was observed to mate once within $20 \mathrm{~min}$ and the mating lasted more than $2 \mathrm{~min}$. If this did not occur, the couple (or at least the male) was discharged. The singlemated female was subsequently placed in a $120-\mathrm{mL}$ glass jar containing 60 cowpea seeds and food source for the adults (a dilute solution of $0.50 \mathrm{~g}$ yeast extract and $8.0 \mathrm{~g}$ pure clear honey dissolved in $40 \mathrm{~mL}$ of deionized and distilled water), which was placed in a 1.5-mL Eppendorf lid containing absorbent cotton wool and daily replenished. Food source for the adult insects was provided because they have access to food source in cowpea fields where the same flightless morph prevails, except during eventual and brief periods of dispersion (Messina \& Renwick, 1985; Ouedraogo et al., 1991). This procedure allows maximum expression of their reproductive potential but minimizes the potential nutritional value of the male ejaculate to the female.

Single-mated females were maintained alone in the jar but the multiple-mated females were placed together with a virgin male, which was daily replaced with another virgin male of the same replicated line for seven consecutive days, after which the female was left alone. Such procedure, although allowing maximum expression of female fecundity, does not control for the extent of monandry and polyandry during the multiple mating by each female, which was focus of previous studies (Eady et al., 2000; Moya-Laraño \& Fox, 2006). The experimental units were daily observed and female mortality was recorded daily until their death. The cowpea seeds were changed daily for five consecutive days (the first 2-3 days are the most fecund), therefore securing the provision of more egg-laying substrates (i.e., seeds) than needed for the uniform distribution of one egg per seed; a trait commonly associated with contest competition strains but not scramble competition strains (Smith \& Lessells, 1985; Smith, 1990; Messina, 1991). Seeds of each replaced batch were inspected for total number of eggs and the number of first-day singleton eggs laid by each female. Egg weight was recorded from 
at least 50 singleton eggs (1 day old) from at least five different females (ten eggs per female; second day of egg-laying). Eggs were removed from the seeds with a scalpel under a stereomicroscope and weighed within $\pm 0.1 \mu \mathrm{g}$.

\section{Statistical analysis}

Data on number of insects emerged per seed used for establishing new generations and female body weight were subjected to analysis of variance (ANOVA) for the parental strains, $F_{1}$ reciprocal crosses and the competition-selected lines after six generations of selection $(P<0.05)$. Data for the parental strains and $F_{1}$ reciprocal crosses were subjected to fixed-model analysis of covariance (ANCOVA) as described below. By contrast, data for the third generation of relaxed selection was subjected to mixed-model ANCOVA with the replicated line as random variable and data for the competitionselected lines after six generations of selection were subjected to mixed-model Ancova with the replicated lines as random variable nested within the competition-selected lines, except for egg weight. Only fixed-models were used for egg weight because the eggs were taken from just five females, which were the replicates for the analyses. Two-way ANCOVA with line and mating type as dependent variable and female weight as covariate was applied for female fecundity and egg weight. The same analysis was also used for female longevity but including female fecundity as covariate. Twoway ANOVA were used to analyse the proportion of first day singleton eggs laid per female. The general linear model was used for these analyses using the procedure PROC GLM from SAS, with the RANDOM statement when appropriate (i.e., for mixed-models) (SAS Institute, 1997). Assumptions of normality and homogeneity of variances were tested using the procedure PROC UNIVARIATE (SAS Institute, 1997), and no data transformation was necessary.

\section{Results}

The crosses and subsequent selection on larval competition strategy carried out succeeded in selecting scramble and contest lines of larval competition (Fig. 1). The number of adults emerging per seed bearing over five eggs in the parental lines was $2.38 \pm 0.03$ and $1.75 \pm 0.02$ (mean $\pm \mathrm{SE}$ ) for the Brazilian and Indian strains, respectively, which were significantly different $\left(F_{1,2}=337.35, P=0.003\right)$. The two $F_{1}$ reciprocal crosses showed indistinguishable outcome of competition with $1.71 \pm 0.03$ adults emerging per seed $\left(F_{1,2}=\right.$ $0.08, P=0.80)$, closely resembling the competition outcome of the Indian strain, $\mathrm{F}_{2}(1.71 \pm 0.01$ adults emerged per seed $)$ and even the relaxed selection for three generations (1.61 \pm 0.09 adults emerged per seed). The selection protocol, however, led to a quick divergence of competition strategies, particularly in the first two generations, leading to $2.84 \pm 0.36$ and $0.68 \pm 0.09$ adults emerged per seed in the scrambleand contest-selected lines after six generations of selection $\left(F_{1,6}=24.28, P=0.02\right)$.

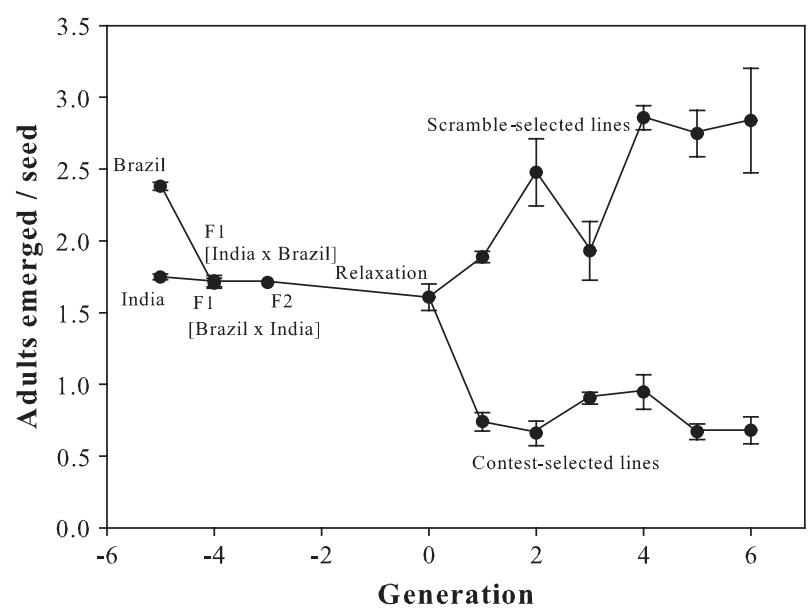

Fig. 1. Number of adults emerged per seed of lines of Callosobruchus maculatus subjected to selection for scramble and contest competition. Vertical bars indicate SE.

\section{Correlated responses of female body weight and fecundity}

The selection of competition strategies led to correlated selection of female body weight (Fig. 2). Females from the Indian strain were significantly heavier than females from the Brazilian strain $(6.64 \pm 0.21 \mathrm{mg}$ and $5.82 \pm 0.09 \mathrm{mg}$, respectively; $\left.F_{1,73}=11.32, P=0.001\right)$. Females from the $F_{1}$ crosses were similar $(6.25 \pm 0.14 \mathrm{mg}$ and $6.52 \pm 0.15 \mathrm{mg}$; $\left.F_{1,74}=1.79, P=0.18\right)$ and resembled the female weight from the relaxed selection regime $(6.24 \pm 0.14 \mathrm{mg})$. Female weights for the competition-selected lines were not significantly different $\left(F_{1,80}=2.79, P=0.09\right)$ but showed the same trend observed for the parental lines with heavier females in the contest-selected lines $(5.77 \pm 0.15 \mathrm{mg}$ for the scrambleselected lines and $6.18 \pm 0.17 \mathrm{mg}$ for the contest-selected

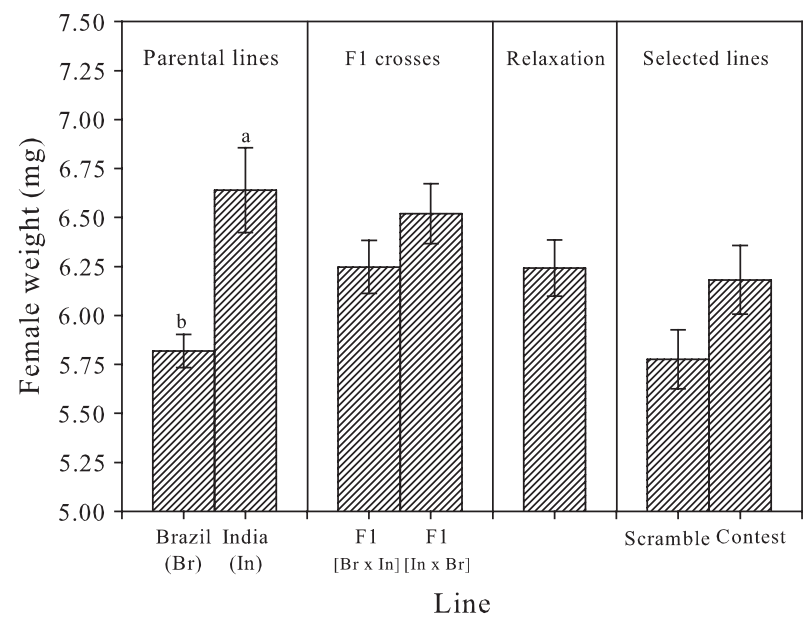

Fig. 2. Female body weight (mg) of lines of Callosobruchus maculatus subjected to selection for scramble and contest competition. Letters above the histogram bars indicate a significant difference between lines (Fisher's $F$-test; $P<0.05$ ). Vertical bars indicate $\mathrm{SE}$. 
lines after six generations of selection). The replicated lines of relaxed selection and of the competition-selected lines were not significantly different for any of the traits investigated $(P>0.05)$, including female weight.

Female weight of $C$. maculatus usually affects fecundity and was therefore used as covariate in the analyses of covariance of female fecundity when the strains from the parental lines, $F_{1}$ crosses, relaxed selection and competition-selected lines were subjected to single and multiple (polyandrous) matings (Tables 1,2). The interaction between line and mating type was not significant $(P>0.05)$, unlike the effects of line and mating type. Female fecundity was strongly affected by female weight at emergence and also by the selected lines, which followed the trend of the parental lines (Tables 1, 2; Fig. 3a). Multiple mating led to higher fecundity in the parental lines, $F_{1}$ crosses and relaxed selection, and was also observed for the competition-selected lines (Tables 1, 2; Fig. 3b).

\section{Correlated responses of female longevity}

Female weight and fecundity may affect female longevity and were therefore used as covariates in the analyses of covariance of the last trait, which proved to be important (Tables 3, 4). Once again, the interaction between line

Table 1. Results of analyses of covariance for female fecundity (total eggs laid per female) of lines of Callosobruchus maculatus subjected to selection for scramble and contest competition outcome and two mating regimes (single and multiple mated females): fixed-model analyses of covariance.

\begin{tabular}{|c|c|c|c|c|c|c|}
\hline \multirow{2}{*}{$\begin{array}{l}\text { Source of } \\
\text { variation }\end{array}$} & \multicolumn{3}{|c|}{$\begin{array}{l}\text { Parental lines (Brazilian } \\
\text { and Indian strains) }\end{array}$} & \multicolumn{3}{|c|}{$F_{1}$ reciprocal crosses } \\
\hline & d.f. & $F$ & $P$ & d.f. & $F$ & $P$ \\
\hline Line & 1 & 12.81 & 0.001 & 1 & 5.73 & 0.02 \\
\hline Mating type & 1 & 28.39 & $<0.001$ & 1 & 20.75 & $<0.001$ \\
\hline $\begin{array}{l}\text { Line } \times \text { mating } \\
\text { type }\end{array}$ & 1 & 0.67 & 0.41 & 1 & 0.01 & 0.91 \\
\hline Female weight & 1 & 21.96 & $<0.001$ & 1 & 4.02 & 0.04 \\
\hline Error & 70 & & & 71 & & \\
\hline
\end{tabular}

and mating type was not significant for any of the comparisons tested [i.e. parental lines, $F_{1}$ crosses, relaxed selection and competition-selected were subjected to single and multiple (polyandrous) mating)], nor was the line (Tables 3 , 4). Multiple mating significantly reduced longevity in the parental lines, as well as in the competition-selected lines (Fig. 4).

Correlated responses of egg weight and egg-laying behaviour

Egg weight and egg-laying behaviour (measured as the number of eggs laid singly on a seed per female during her first day of egg laying or number of first day singleton eggs laid) results of parental lines, $F_{1}$ crosses, relaxed selection and competition-selected lines for six generations were subjected to either two-way ANCOVA with female weight as covariate (egg weight) or two-way ANOvA (proportion of singleton eggs) to test the effects of line and mating type (except for relaxed selection where there was no line effect). The interactions between line and mating type were not significant for any of the traits assessed (Tables 5-7). Eggs from females of the Indian strain were significantly heavier than eggs from the Brazilian strain; the same trend was not significant $(P>0.05)$ in the competition-selected lines (Table 5; Fig. 5). Mating type did not affect egg weight (Table 5).

The mating type did not affect the female egg-laying behaviour through the proportion of first day singleton eggs laid (Tables 6, 7). Only line showed a significant effect on the proportion of singleton eggs laid with the Indian strain and the contest-selected lines laying a higher proportion of singleton eggs than the Brazilian strain and the scrambleselected lines, respectively (Fig. 6).

\section{Discussion}

It is expected that selection based on larval-competition strategy would produce a correlated response to exposure to multiple (polyandrous) matings. Specifically, scramble-selected lines would evolve a response to polyandry of increased fecundity and reduced longevity but contest-selected lines would

Table 2. Results of analyses of covariance for female fecundity (total eggs laid per female) of lines of Callosobruchus maculatus subjected to selection for scramble and contest competition outcome and two mating regimes (single and multiple mated females): mixed-model (nested for selected lines) analysis of variance.

\begin{tabular}{|c|c|c|c|c|c|c|c|c|}
\hline \multirow{2}{*}{$\begin{array}{l}\text { Source of } \\
\text { variation }\end{array}$} & \multicolumn{4}{|c|}{ Relaxation period } & \multicolumn{4}{|c|}{ Selected lines for scramble and contest competition } \\
\hline & d.f. & d.f. ${ }_{\text {error }}$ & $F$ & $P$ & d.f. & d.f. ${ }_{\text {error }}$ & $F$ & $P$ \\
\hline Line & - & & & & 1 & 6.63 & 31.36 & 0.001 \\
\hline $\begin{array}{l}\text { Replicated line or replicated line } \\
\text { (line) }\end{array}$ & 3 & 2.90 & 1.13 & 0.46 & 6 & 72 & 0.99 & 0.44 \\
\hline Mating type & 1 & 3.46 & 8.04 & 0.05 & 1 & 72 & 3.58 & 0.05 \\
\hline Line $\times$ mating type & - & & & & 1 & 72 & 1.90 & 0.17 \\
\hline Replicated line $\times$ mating type & 3 & 28 & 0.79 & 0.51 & - & & & \\
\hline Female weight & 1 & 28 & 10.54 & 0.003 & 1 & 72 & 57.20 & $<0.001$ \\
\hline
\end{tabular}


(a)

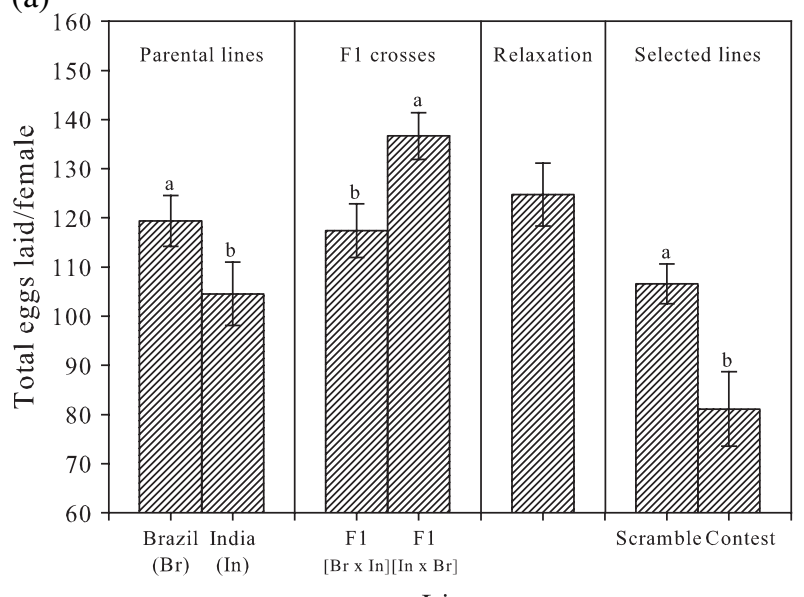

Line

(b)

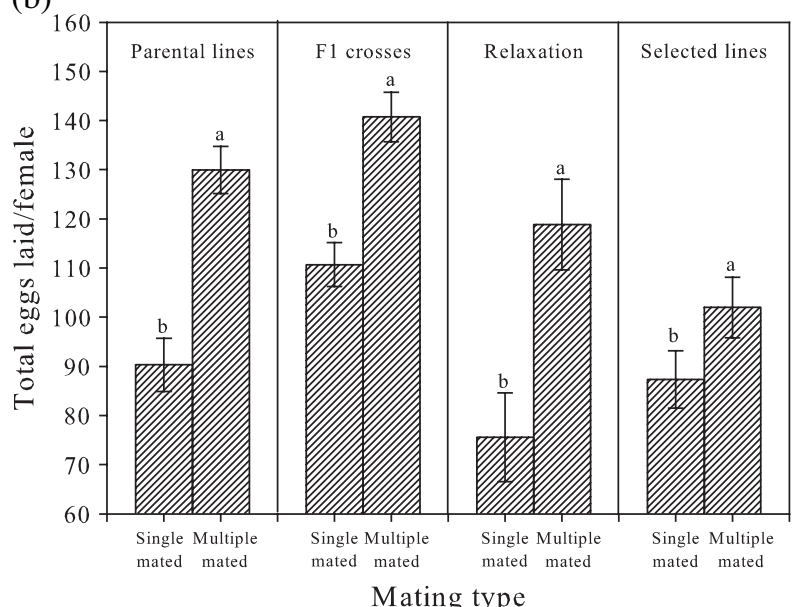

Fig. 3. Female fecundity (total eggs laid per female) of lines of Callosobruchus maculatus subjected to selection for scramble and contest competition outcome and two mating regimes (single- and multiple-mated females). Letters above the histogram bars of different lines (a) or mating type (b) indicate a significant difference (Fisher's $F$-test; $P<0.05$ ). Vertical bars indicate SE.
Table 3. Results of analyses of covariance for female longevity (days) of lines of Callosobruchus maculatus subjected to selection for scramble and contest competition outcome and two mating regimes (single and multiple mated females): fixed-model analyses of covariance.

\begin{tabular}{|c|c|c|c|c|c|c|}
\hline \multirow[b]{2}{*}{ Source of variation } & \multicolumn{3}{|c|}{$\begin{array}{l}\text { Parental lines (Brazilian } \\
\text { and Indian strains) }\end{array}$} & \multicolumn{3}{|c|}{$\mathrm{F}_{1}$ reciprocal crosses } \\
\hline & d.f. & $F$ & $P$ & d.f. & $F$ & $P$ \\
\hline Line & 1 & 1.34 & 0.25 & 1 & 0.61 & 0.44 \\
\hline Mating type & 1 & 5.52 & 0.02 & 1 & 3.06 & 0.07 \\
\hline Line $\times$ mating type & 1 & 0.20 & 0.66 & 1 & 1.89 & 0.17 \\
\hline Female weight & 1 & 0.57 & 0.45 & 1 & 4.69 & 0.03 \\
\hline Eggs & 1 & 3.39 & 0.07 & 1 & 47.28 & $<0.001$ \\
\hline Error & 69 & & & 71 & & \\
\hline
\end{tabular}

not respond to polyandry. Instead, both scramble- and contestselected lines show increased fecundity and reduced longevity with polyandry, which does not affect egg weight. Female egg-laying behaviour is not at all affected by polyandry, only by line and competition strategy as expected based on previous studies (Smith \& Lessells, 1985; Messina, 1991).

The scramble-selected lines follow the expected prediction showing increased fecundity and reduced longevity with polyandry. The trade-off between fecundity and longevity in the Brazilian strain subjected to polyandry is also reported by Lobley-Taylor (2000) when assessing costs and benefits of multiple mating in $C$. maculatus. Females of the Brazilian strain display phenotypic plasticity in their ability to trade fecundity and longevity depending on the availability of egglaying sites; they are able to maximize egg laying when seeds are abundant but trade fecundity for longevity when seeds are poor or limited (Messina \& Slade, 1999; Messina \& Fry, 2003). In the present study, an abundance of seeds is provided for egg-laying, besides a complementary food source for adults, to maximize fecundity. Furthermore, multiple (polyandrous) mating provides not only enough gametes for maximum offspring production, a likely contrast with single mated females but also ejaculates that appear to have a double role of egg-laying stimulant and nutrient provision

Table 4. Results of analyses of covariance for female longevity (days) of lines of Callosobruchus maculatus subjected to selection for scramble and contest competition outcome and two mating regimes (single and multiple mated females): mixed-model (nested for selected lines) analysis of variance.

\begin{tabular}{|c|c|c|c|c|c|c|c|c|}
\hline \multirow[b]{2}{*}{ Source of variation } & \multicolumn{4}{|c|}{ Relaxation period } & \multicolumn{4}{|c|}{ Selected lines for scramble and contest competition } \\
\hline & d.f. & d.f. & $F$ & $P$ & d.f. & d.f. $_{\text {error }}$ & $F$ & $P$ \\
\hline Line & - & & & & 1 & 11.05 & 0.00 & 0.96 \\
\hline $\begin{array}{l}\text { Replicated line or replicated } \\
\text { line(strain) }\end{array}$ & 3 & 2.69 & 1.94 & 0.31 & 6 & 71 & 1.35 & 0.25 \\
\hline Mating type & 1 & 6.79 & 0.63 & 0.46 & 1 & 71 & 11.83 & 0.001 \\
\hline Line $\times$ mating type & - & & & & 1 & 71 & 0.48 & 0.49 \\
\hline Replicated line $\times$ mating type & 3 & 27 & 0.52 & 0.67 & - & & & \\
\hline Female weight & 1 & 27 & 0.72 & 0.40 & 1 & 71 & 7.26 & 0.001 \\
\hline Eggs & 1 & 27 & 4.18 & 0.06 & 1 & 71 & 52.36 & $<0.001$ \\
\hline
\end{tabular}

(C) 2008 The Authors

Journal compilation () 2008 The Royal Entomological Society, Physiological Entomology, 33, 372-381 


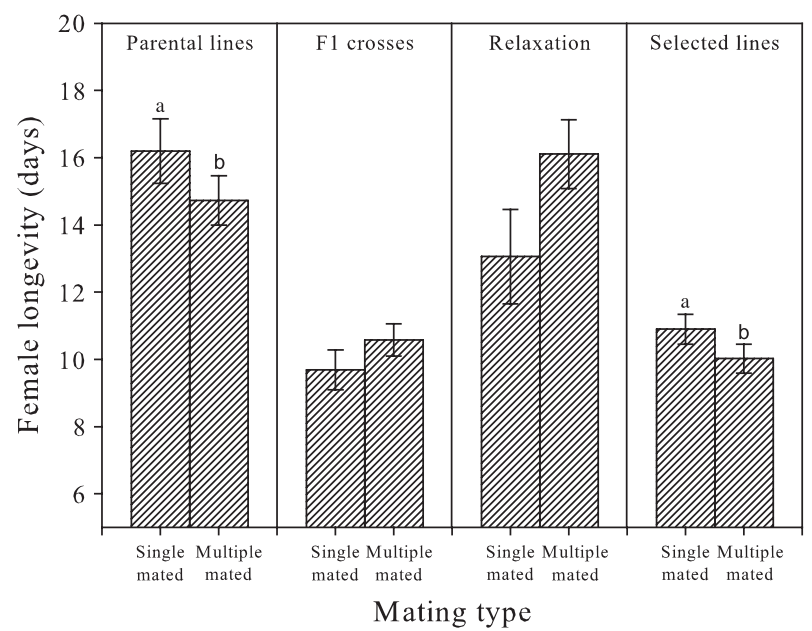

Fig. 4. Female longevity (days) of lines of Callosobruchus maculatus subjected to selection for scramble and contest competition outcome and two mating regimes (single- and multiple-mated females). Letters above the histogram bars indicate a significant difference between mating type (Fisher's $F$-test; $P<0.05$ ). Vertical bars indicate $\mathrm{SE}$.

(Savalli \& Fox, 1999; Wilson et al., 1999; Paukku \& Kotiaho, 2005; Yamane \& Miyatake, 2005). The potential nutritional role of the ejaculate is largely denied by the provision of a complementary food source for adults, which is also available for them in the field when foraging in seeds of living plants, as is the case for C. maculatus. Despite this, increased fecundity still takes place under multiple (polyandrous) mating in all strains and lines assessed, as compared with when they are single mated.

The provision of male ejaculates as nuptial gifts for females is a direct benefit of multiple mating (Vahed, 1998; Hosken \& Stockley, 2003). Callosobruchus maculatus is reported to produce and transfer large ejaculates during mating (Fox, 1993; Fox et al., 1995; Yamane \& Miyatake, 2005). However, the way that females benefit from the male ejaculate appears to vary in $C$. maculatus because there are reports on extended longevity and the opposite effect as well, depending on strain, male density and mating status (Fox, 1993; Savalli \& Fox, 1999; Paukku \& Kotiaho, 2005; Yamane \& Miyatake, 2005). Because the fecundity-longevity trade-off is reported in the present study, where strain, male density and mating status are controlled and additional nutrient source is provided, the stimulatory role of cowpea weevil ejaculate probably prevails over the nutritional role, as expected based on Wilson et al. (1999). The lack of differences in egg weight from single and multiple mating reinforces this suggestion because a prevailing nutritional role of the ejaculate would probably lead to the production of heavier eggs when the females are mated multiply.

The multiple mating benefits in the present study are unable to compensate the costs of reproduction, represented in C. maculatus by the cost of mating (collateral harm by genital damage, minimized by female kicking), male harassment and cost of egg production (Crudgington \& Siva-Jothy, 2000; Morrow et al., 2003; Yanagi \& Miyatake, 2003; Edvardsson \& Tregenza, 2005; Paukku \& Kotiaho, 2005; Rönn et al., 2006). The more fecund females from the Brazilian strain and the scramble-selected lines incur higher costs of egg production and a compromise in longevity is expected and observed. Collateral harm by female genital damage may also increase with multiple mating but female kicking significantly minimizes this cost (Crudgington \& Siva-Jothy, 2000). Male harassment is maximal under multiple polyandrous mating with virgin males and non-existent for single mated females, further compromising female longevity in the former case. The higher fecundity is a trait associated with the scramble competition strategy observed in C. maculatus and the higher fecundity of the scramble-selected lines is therefore expected.

The lack of a correlated response between female fecundity and longevity expected in the Indian strain and the contestselected lines by exposure to polyandry is not observed. Both strains show the same correlated response that is observed (and predicted) for the Brazilian strain and the scramble-selected lines. The Indian strain is a contest competitor and the females are more discriminating in their egglaying behaviour, showing a more uniform egg distribution than the Brazilian strain (e.g. Smith \& Lessells, 1985; Messina, 1991; Lobley-Taylor, 2000), which is also observed in the present study. Therefore, selection on Indian females is for extended longevity rather than fecundity, so that females live long enough to enable them to seek out sufficient good quality sites for egg-laying, thus reducing potential fecundity in favour of realized fecundity (Lobley-Taylor, 2000).

Table 5. Results of analyses of covariance for egg weight ( $\mu \mathrm{g})$ of lines of Callosobruchus maculatus subjected to selection for scramble and contest competition outcome and two mating regimes (single and multiple mated females).

\begin{tabular}{|c|c|c|c|c|c|c|c|c|c|c|c|c|}
\hline \multirow[b]{2}{*}{ Source of variation } & \multicolumn{3}{|c|}{$\begin{array}{l}\text { Parental lines (Brazilian and } \\
\text { Indian strains) }\end{array}$} & \multicolumn{3}{|c|}{$F_{1}$ reciprocal crosses } & \multicolumn{3}{|c|}{ Relaxation period } & \multicolumn{3}{|c|}{$\begin{array}{l}\text { Selected lines for scramble } \\
\text { and contest competition }\end{array}$} \\
\hline & d.f. & $F$ & $P$ & d.f. & $F$ & $P$ & d.f. & $F$ & $P$ & d.f. & $F$ & $P$ \\
\hline Line & 1 & 6.24 & 0.02 & 1 & 1.78 & 0.19 & - & & & 1 & 2.53 & 0.13 \\
\hline Mating type & 1 & 0.22 & 0.65 & 1 & 1.36 & 0.26 & 1 & 3.95 & 0.07 & 1 & 3.25 & 0.09 \\
\hline Line $\times$ mating type & 1 & 0.87 & 0.36 & 1 & 0.33 & 0.58 & - & & & 1 & 0.14 & 0.72 \\
\hline Female weight & 1 & 4.67 & 0.04 & 1 & 1.40 & 0.25 & 1 & 7.03 & 0.03 & 1 & 0.78 & 0.39 \\
\hline Error & 15 & & & 15 & & & 7 & & & 15 & & \\
\hline
\end{tabular}


Table 6. Results of analysis of variance for proportion of first day singleton eggs laid per female of lines of Callosobruchus maculatus subjected to selection for scramble and contest competition outcome and two mating regimes (single and multiple-mated females): fixed-model analyses of covariance.

\begin{tabular}{lcccccccc}
\hline & \multicolumn{2}{l}{$\begin{array}{l}\text { Parental lines (Brazilian } \\
\text { and Indian strains) }\end{array}$} & & \multicolumn{2}{l}{$F_{1}$ reciprocal crosses } \\
\cline { 2 - 4 } Source of variation & d.f. & $F$ & $P$ & & d.f. & $F$ & $P$ \\
\hline Line & 1 & 4.52 & 0.04 & & 1 & 1.81 & 0.18 \\
Mating type & 1 & 0.40 & 0.53 & & 1 & 0.47 & 0.49 \\
Line $\times$ mating type & 1 & 0.26 & 0.61 & & 1 & 0.83 & 0.37 \\
Error & 71 & & & & 72 & & \\
\hline
\end{tabular}

Polyandry in Indian females may lead to excess ejaculates that could increase egg-laying stimulation, egg size (and thus progeny quality) due to derived nutrients, or both. However, because insects from India exhibit contest-competition with high juvenile mortality, the expectation is that polyandry would increase egg weight but would not have any impact on female fecundity and longevity of the contest-selected lines.

The Indian strain also shows increased fecundity and reduced longevity with multiple (polyandrous) matings, similar to the Brazilian strain. In addition, egg weight is not affected by multiple (polyandrous) mating. Therefore the expected between-population variation in the resolution of sexual conflict in $C$. maculatus is not observed, unlike in a preliminary investigation with the parental strains (i.e. Brazilian and Indian) (Lobley-Taylor, 2000). Arbitrary divergence between the parental colonies used in the present study and those of Lobley-Taylor (2000) may have occurred during the interval between both studies as recently demonstrated (Fricke \& Arnqvist, 2004). Regardless of that, the contest competition Indian strain remains as such but the expected (or rather lack of) fecundity-longevity trade-off under multiple (polyandrous) mating does not occur. This apparent sub-optimal

Table 7. Results of analysis of variance for proportion of first day singleton eggs laid per female of lines of Callosobruchus maculatus subjected to selection for scramble and contest competition outcome and two mating regimes (single and multiple-mated females): mixed-model (nested for selected lines) analysis of variance.

\begin{tabular}{|c|c|c|c|c|c|c|c|c|}
\hline \multirow{2}{*}{$\begin{array}{l}\text { Source of } \\
\text { variation }\end{array}$} & \multicolumn{4}{|c|}{ Relaxation period } & \multicolumn{4}{|c|}{$\begin{array}{l}\text { Selected lines for scramble } \\
\text { and contest competition }\end{array}$} \\
\hline & d.f. & d.f. $_{\text {error }}$ & $F$ & $P$ & d.f. & d.f. $_{\text {error }}$ & $F$ & $P$ \\
\hline Line & - & & & & 1 & 73 & 5.76 & 0.04 \\
\hline $\begin{array}{l}\text { Replicated line } \\
\text { or replicated } \\
\text { line(strain) }\end{array}$ & 3 & 3 & 3.14 & 0.19 & 6 & 6.15 & 1.38 & 0.23 \\
\hline Mating type & 1 & 3.06 & 0.68 & 0.47 & 1 & 73 & 2.75 & 0.10 \\
\hline $\begin{array}{l}\text { Line } \times \\
\text { mating type }\end{array}$ & - & & & & 1 & 73 & 0.17 & 0.69 \\
\hline $\begin{array}{l}\text { Replicated line } \\
\times \text { mating type }\end{array}$ & 3 & 29 & 0.58 & 0.63 & - & & & \\
\hline
\end{tabular}

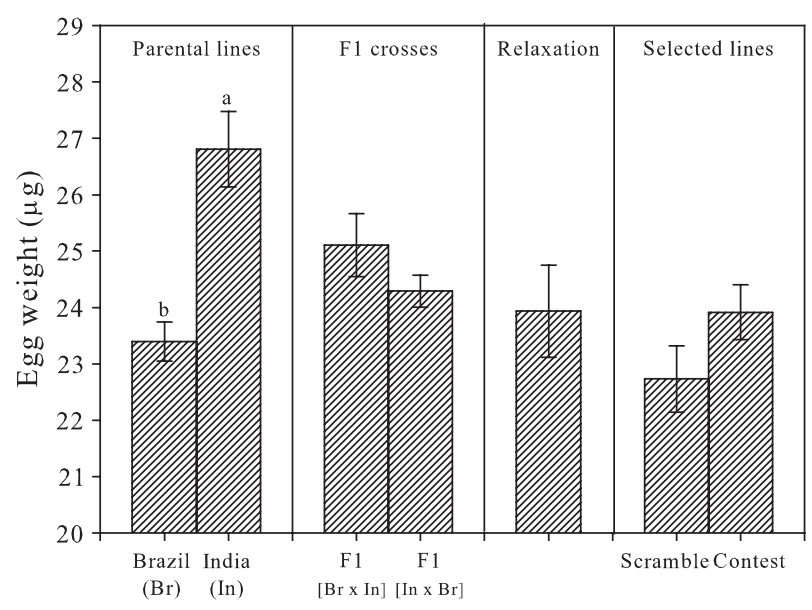

Line

Fig. 5. Egg weight $(\mu \mathrm{g})$ of lines of Callosobruchus maculatus subjected to selection for scramble and contest competition outcome and two mating regimes (single- and multiple-mated females). Letters above the histogram bars indicate a significant difference between lines (Fisher's $F$-test; $P<0.05$ ). Vertical bars indicate SE.

female behaviour in lines exhibiting larval contest competition is suggestive of the importance of sexual conflict prevailing over larval competition (and therefore survival), and the selection pressure established in the present investigation.

Increased fecundity with the characteristic low juvenile survival of the contest competition is counter-intuitive based on direct fitness benefits for females. However, indirect benefits may be playing a relevant role in resolving such conflicting outcome in contest-competition lines despite being currently regarded as of secondary importance in resolving

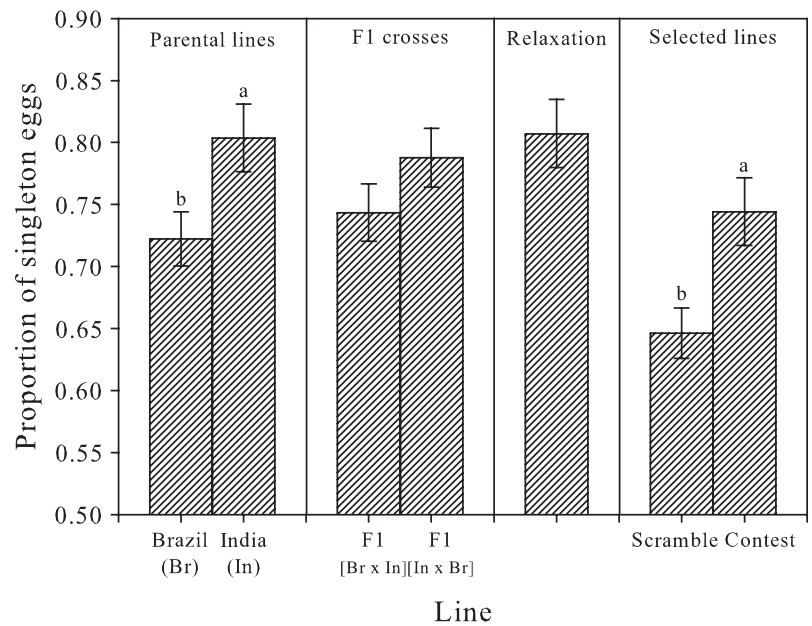

Fig. 6. Proportion of first day singleton eggs laid per female of lines of Callosobruchus maculatus subjected to selection for scramble and contest competition outcome and two mating regimes (single and multiple mated females). Letters above the histogram bars indicate a significant difference between lines (Fisher's $F$-test; $P<0.05$ ). Vertical bars indicate SE. 
sexual conflict, particularly when considering multiple (polyandrous) mating as in the present study (Arnqvist \& Nilsson, 2000; Hosken \& Stockley, 2003; Tregenza et al., 2006). Increased offspring diversity and increased offspring fitness are the indirect (genetic) benefits that may play a relevant role in determining the fecundity-longevity trade-off for contest competitors leading to increased fecundity in lines showing high juvenile mortality (Jennions \& Petrie, 2000; Hosken \& Stockley, 2003).

The increased fecundity achieved with multiple (polyandrous) mating will increase the genetic diversity of the offspring. The genetically diverse range of offspring increases the likelihood that some of them will possess viability genes that enable them to survive and breed in the next generation, particularly in heterogeneous or unpredictable environments (Yasui, 1998; Jennions \& Petrie, 2000). In addition, polyandrous females with increased fecundity can elevate offspring fitness by increasing the number of potential sires that compete (Jennions \& Petrie, 2000; Hosken \& Stockley, 2003). The fierce competition within the grain with active interference between juveniles will impose strong selection pressure favouring the survival of the fittest among contest competitors of C. maculatus leading to an increase in offspring quality rather than quantity. These two possibile indirect benefits potentially explain the unexpected combination of increased fecundity coupled with high juvenile mortality that is observed in the contest competition lines of $C$. maculatus in the present study. Density-dependence is assumed to affect mating systems, despite the clear need for further work examining the interaction between population ecology (specifically density) and sexual conflict (Kokko \& Rankin, 2006; Tregenza et al., 2006). This belief is based on adult competition studies (Kokko \& Rankin, 2006) but juvenile competition may be as important at least in some taxa, as suggested by our argument, and the subject is worthy of future attention.

\section{Acknowledgements}

We thank the U.K. Natural Resources Institute, Dr Peter Credland and Dr Frank Messina for providing the original stock strains of $C$. maculatus used in the present study. Financial support provided by the CAPES Foundation (Brazilian Ministry of Education) and the National Council of Scientific and Technological Development (Brazilian Ministry of Science and Technology) is greatly appreciated. The comments and suggestions provided by Professor J. Hardie and two anonymous referees are also greatly appreciated.

\section{References}

Arnqvist, G. \& Nilsson, T. (2000) The evolution of polyandry: multiple mating and female fitness in insects. Animal Behaviour, 60, $145-164$.

Arnqvist, G., Nilsson, T. \& Katvala, M. (2005) Mating rate and fitness in female bean weevils. Behavioral Ecology, 16, 123-127.
Broadhurst, L.J. (1996) The evolution of larval competition and oviposition behaviour in the cowpea weevil Callosobruchus maculatus (Fab.) (Coleoptera: Bruchidae). PhD Dissertation, University of Leicester, U.K.

Crudgington, H.S. \& Siva-Jothy, M.T. (2000) Genital damage, kicking and early death. Nature, 407, 855-856.

Eady, P.E., Wilson, N. \& Jackson, M. (2000) Copulating with multiple mates enhances female fecundity but not egg-to-adult survival in the bruchid beetle Callosobruchus maculates. Evolution, 54, 2161-2165.

Edvardsson, M. \& Tregenza, T. (2005) Why do male Callosobruchus maculatus harm their mates? Behavioral Ecology, 16, 788-793.

Fox, C.W. (1993) Multiple mating, lifetime fecundity and female mortality of the bruchid beetle, Callosobruchus maculatus. Functional Ecology, 7, 203-208.

Fox, C.W., Hickman, D.L., Raleigh, E.L. \& Mousseau, T.A. (1995) Paternal investiment in a seed beetle (Coleoptera: Bruchidae): influence of male size, age, and mating history. Annals of the Entomological Society of America, 88, 100-103.

Fricke, C. \& Arnqvist, G. (2004) Divergence in replicated phylogenies: the evolution of partial post-mating prezygotic isolation in bean weevils. Journal of Evolutionary Biology, 17, 1345-1354.

Guedes, R.N.C., Smith, R.H. \& Guedes, N.M.P. (2003) Host suitability, respiration rate and the outcome of larval competition in strains of the cowpea weevil, Callosobruchus maculatus. Physiological Entomology, 28, 298-305.

Guedes, R.N.C., Guedes, N.M.P. \& Smith, R.H. (2007) Larval competition within seeds: from the behavioural process to the ecological outcome in the seed beetle Callosobruchus maculatus. Austral Ecology, 32, 697-707.

Hosken, D.J. \& Stockley, P. (2003) Benefits of polyandry: a life history perspective. Evolutionary Biology, 33, 173-194.

Jennions, M.D. \& Petrie, M. (2000) Why do females mate multiply? A review of the genetic benefits. Biological Reviews, 75, 21-64.

Johnstone, R.A. \& Keller, L. (2000) How males can gain by harming their mates: sexual conflict, seminal toxins, and the cost of mating. American Naturalist, 156, 368-377.

Kokko, H. \& Rankin, D.J. (2006) Lonely hearts or sex in the city? Density-dependent effects in mating systems. Philosophical Transactions of the Royal Society of London Series B, 361, 319-334.

Lessells, C.M. (2005) Why are males bad for females? Models for the evolution of damaging male mating behavior. American Naturalist, 165, S46-S63.

Lessells, C.M. (2006) The evolutionary outcome of sexual conflict. Philosophical Transactions of the Royal Society of London Series $B, \mathbf{3 6 1}, 301-317$.

Lobley-Taylor, T.J.B. (2000) Costs and benefits of multiple mating in the beetle Callosobruchus maculatus (F.) (Coleoptera: Bruchidae). PhD Dissertation, University of Leicester, U.K.

Messina, F.J. (1991) Life-history variation in a beetle: adult egglaying vs. larval competition ability. Oecologia, 85, 447-455.

Messina, F.J. (2004) Predictable modification of body size and competitive ability following a host shift by a seed beetle. Evolution, 58, 2788-2797.

Messina, F.J. \& Fry, J.D. (2003) Environment-dependent reversal of a life-history trade-off in the seed beetle Callosobruchus maculatus. Journal of Evolutionary Biology, 16, 501-509.

Messina, F.J. \& Renwick, J.A.A. (1985) Dispersal polymorphism of Callosobruchus maculates (Coleoptera: Bruchidae): variation among populations in response to crowding. Annals of the Entomological Society of America, 78, 201-206. 
Messina, F.J. \& Slade, A.F. (1999) Expression of a life-history tradeoff in a seed beetle depends on environmental context. Physiological Entomology, 24, 358-363.

Morrow, E.H., Arnqvist, G. \& Pitnick, S. (2003) Adaptation versus pleiotropy: why do males harm their mates? Behavioral Ecology, 14, 802-806.

Moya-Laraño, J. \& Fox, C.W. (2006) Ejaculate size, second male size, and moderate polyandry increase female fecundity in a seed beetle. Behavioral Ecology, 17, 940-946.

Nicholson, A.J. (1954) An outline of the dynamics of animal populations. Australian Journal of Zoology, 2, 9-65.

Ouedraogo, P.A., Monge, J.P. \& Huignard, J. (1991) Importance of temperature and seed water content on the induction of imaginal polymorphism in Callosobruchus maculatus. Entomologia Experimentalis et Applicata, 59, 59-66.

Parker, G.A. (1979) Sexual selection and sexual conflict. Sexual Selection and Reproductive Competition in Insects (ed. by M. S. Blum and A. N. Blum), pp. 123-166. Academic, U.K.

Parker, G.A. (2006) Sexual conflict over mating and fertilization: an overview. Philosophical Transactions of the Royal Society of London Series B, 361, 235-259.

Paukku, S. \& Kotiaho, J.S. (2005) Cost of reproduction in Callosobruchus maculatus: effects of mating on male longevity and the effect of male mating status on female longevity. Journal of Insect Physiology, 51, 1220-1226.

Rönn, J., Katvala, M. \& Arnqvist, G. (2006) The costs of mating and egg production in Callosobruchus seed beetles. Animal Behaviour, 72, 335-342.

SAS Institute (1997) SAS/STAT User's Guide, Version 8. SAS Institute, Cary, North Carolina.

Savalli, U.M. \& Fox, C.W. (1999) The effect of male mating history on paternal investment, fecundity and female remating in the seed beetle Callosobruchus maculatus. Functional Ecology, 13, 169-177.

Sibly, R.M. \& Calow, P. (1986) Physiological Ecology of Animals: An Evolutionary Approach. Blackwell, U.K.
Smith, R.H. (1990) Adaptations of Callosobruchus maculatus to competition. Bruchids and Legumes: Economics, Ecology and Coevolution (ed. by K. Fujii, A. M. R. Gatehouse, C. D. Johnson, R. Mitchell and Y. Yoshida), pp. 351-360, Kluwer, The Netherlands.

Smith, R.H. (1991) Genetic and phenotypic aspects of life-history evolution in animals. Advances in Ecological Research, 21, 63-120.

Smith, R.H. \& Lessells, C.M. (1985). Oviposition, ovicide and larval competition in granivorous insects. Behavioural Ecology: Ecological Consequences of Adaptative Behaviour (ed. by R. M. Sibly and R. H. Smith), pp. 423-488. Blackwell, U.K.

Tran, B.M.D. \& Credland, P.F. (1995) Consequences of inbreeding for the cowpea seed beetle, Callosobuchus maculatus (F.) (Coleoptera: Bruchidae). Biological Journal of the Linnean Society, $\mathbf{5 6}$ 483-503.

Tregenza, T., Wedell, N. \& Chapman, T. (2006) Sexual conflict: a new paradigm? Philosophical Transactions of the Royal Society of London Series B, 361, 229-234.

Vahed, K. (1998) The function of nuptial feeding in insects: a review of empirical studies. Biological Reviews, 73, 43-78.

Wilson, N., Tufton, T.J. \& Eady, P.E. (1999) The effect of single, double, and triple matings on the lifetime fecundity of Callosobruchus analis and Callosobruchus maculatus (Coleoptera: Bruchidae). Journal of Insect Behavior, 12, 295-306.

Yamane, T. \& Miyatake, T. (2005) Intra-specific variation in strategic ejaculation according to level of polyandry in Callosobruchus maculatus. Journal of Insect Physiology, 51, 1240-1243.

Yanagi, S. \& Miyatake, T. (2003) Costs of mating and egg production in female Callosobruchus chinensis. Journal of Insect Physiology, 49, 823-827.

Yasui, Y. (1998) The 'genetic benefits' of female multiple mating reconsidered. Trends in Ecology and Evolution, 13, 246-250.

Accepted 18 July 2008

First published online 3 October 2008 